\title{
Development of Zr-loaded Green-emitting Liquid Scintillator for Detection of Neutrinoless Double Beta Decay
}

\author{
Akito Watanabe, ${ }^{*}$ Arisa Magi, Masanori Koshimizu, \\ Atsushi Sato, Yutaka Fujimoto, and Keisuke Asai \\ Department of Applied Chemistry, Graduate School of Engineering, Tohoku University, \\ 6-6-07 Aoba, Aramaki, Aoba-ku, Sendai 980-8579, Japan
}

(Received March 6, 2021; accepted May 14, 2021)

Keywords: neutrinoless double beta decay, liquid scintillator, organometallic complex, ${ }^{96} \mathrm{Zr}$

In this study, zirconium acetylacetonate $\left(\mathrm{Zr}(\mathrm{acac})_{4}\right)$ containing the isotope ${ }^{96} \mathrm{Zr}$ was studied as a liquid scintillator for the possible detection of neutrinoless double beta decay. To avoid the absorption of scintillation by ligand absorption, the phosphor Coumarin 7 was assessed as a green-emitting liquid scintillator. By dissolving $3 \mathrm{~g} / \mathrm{L}$ of Coumarin 7 in anisole, a liquid scintillator with a light yield of 12000 photons/MeV was fabricated. Subsequently, the liquid scintillator was loaded with $\mathrm{Zr}(\mathrm{acac})_{4}$. In the liquid scintillator containing $10 \mathrm{wt} \% \mathrm{Zr}(\mathrm{acac})_{4}$, the light yield of scintillation was 370 photons $/ \mathrm{MeV}$ for a blue-emitting liquid scintillator, while a light yield of 1900 photons/MeV was determined for a green-emitting liquid scintillator. The scintillation decay time constants of the fast component were around $4.4 \mathrm{~ns}$. Liquid scintillators with a high light yield that avoid absorption by $\mathrm{Zr}(\mathrm{acac})_{4}$ can be prepared using Coumarin 7 .

\section{Introduction}

Since the discovery of neutrino oscillations, the detection of neutrinoless double beta decay $(0 v \beta \beta)$ has become a research topic in modern particle physics to confirm the mass scale and Majorana nature of neutrinos. ${ }^{(1-3)}$ In $0 v \beta \beta$ events, an even-even nucleus transforms into a lighter isobar containing two more protons with the emission of two electrons and no neutrinos. Since all of the energy of beta decay is given to the two electrons in $0 v \beta \beta$ events, it is expected that distinctive peaks can be detected at the Q-value of the decay in the energy spectrum. In contrast, in a normal double $\beta$ decay event with the emission of two neutrinos, the total energy of the two electrons decreases because part of the decay energy is given to the neutrinos. Hence, the two decay events can be discriminated from the difference in the total energy of the detected electrons. However, the presence of $0 v \beta \beta$ has not been confirmed because the half-life of $0 v \beta \beta$ is extremely long ( $>10^{22}$ years), ${ }^{(4)}$ so only a small number of signals can be expected per year. One approach to detecting events is to use a detector containing hundreds of kilograms of the candidate isotopes of $0 v \beta \beta$.

*Corresponding author: e-mail: evhx92fk4.aki.02@gmail.com https://doi.org/10.18494/SAM.2021.3411 
Liquid scintillation detectors loaded with the candidate isotopes have been widely developed. ${ }^{(5-9)}$ Liquid scintillators are used in the measurement of beta-emitting radioactive nuclides, because by dissolving the radioactive isotopes inside the liquid scintillators, lowenergy beta rays are detected with high efficiency. ${ }^{(10)}$ Therefore, it is expected that $0 v \beta \beta$ events can be detected in a similar usage.

Liquid scintillators convert the energy of irradiated ionizing radiation into visible light, and aromatic organic molecules, particularly benzene derivatives such as toluene and xylene, are widely used as solvents in liquid scintillators. In the design of liquid scintillators, one or two types of phosphor molecules are added to match the emission wavelength with the sensitive region of the photomultiplier tube. To detect an extremely small number of $0 v \beta \beta$ events, the candidate isotopes must be included in the solvents at high concentrations without significantly degrading the optical properties of the liquid scintillator. Various methods for incorporating the candidate isotopes into liquid scintillators have been reported. ${ }^{(5)}$

Several candidate-isotope-loaded liquid scintillators have been prepared by dissolving metal salt compounds containing the candidate isotope; ${ }^{(7,11)}$ however, the solubilities of the metal salt compounds in solvents are generally very low, and loading those compounds leads to quenching of the scintillation. The loading of metals into scintillators is generally through the use of organometallic complexes that are soluble in the liquid scintillators. Organometallic complexes that use a carboxylic acid as a ligand are commonly applied in neutrino experiments. ${ }^{(12-14)} \mathrm{On}$ the other hand, as an alternative to carboxylic acid complexes, beta-diketone complexes are attractive, because they can be expected to have higher chemical stability than carboxylic acids. Furthermore, complexes coordinated with acetylacetone groups have a high solubility in anisole, which is used as a solvent for liquid scintillators. ${ }^{(6,15)}$ Thus, beta-diketone complexes can allow candidate isotopes to be loaded into liquid scintillators at high concentrations.

To date, liquid scintillators containing zirconium beta-diketone complexes have been developed to load ${ }^{96} \mathrm{Zr}$, which is a candidate isotope of $0 v \beta \beta \cdot{ }^{(6,16)}$ The solubility of zirconium acetylacetonate $\left(\mathrm{Zr}(\mathrm{acac})_{4}\right)$ in anisole is known to be more than $10 \mathrm{wt} \%$, while the addition of the complex significantly reduces the scintillation light yield. ${ }^{(6)}$ Some acetylacetone complexes have an absorption tail up to around $400 \mathrm{~nm} .{ }^{(17)}$ Thus, in a typical blue-emitting liquid scintillator, the reduction in the light yield is considered to be due to the absorption of emission by $\operatorname{Zr}(\mathrm{acac})_{4}$.

To suppress the absorption of the emission due to $\mathrm{Zr}(\mathrm{acac})_{4}$, a liquid scintillator that emits at a longer wavelength can be proposed. In this study, 3-(2-benzimidazolyl)-7-(diethylamino) coumarin (Coumarin 7), which exhibits green emission, was used as the phosphor. Coumarin 7 is an amino-substituted coumarin derivative. Since amino-substituted coumarin derivatives exhibit a Stokes shift that strongly depends on the solvent polarity and a high emission quantum yield, many studies on their photophysical properties have been reported. ${ }^{(18-21)}$ On the other hand, since Coumarin 7 has not been used as a phosphor in liquid scintillators, optimization of the concentration is necessary. In this study, the concentration was first optimized. Subsequently, $\mathrm{Zr}(\mathrm{acac})_{4}$ was added to liquid scintillators. The optical and radiation response properties of liquid scintillators containing different concentrations of $\operatorname{Zr}(\text { acac })_{4}$ were investigated. 


\section{Experiments}

Anisole was purchased from FUJIFILM Wako Pure Chemical Co. Ltd. $\operatorname{Zr}(\mathrm{acac})_{4}$ and Coumarin 7 was purchased from Tokyo Chemical Industry Co. Ltd. DPO (2,5-diphenyloxazole) and POPOP (1,4-Bis(5-phenyl-2-oxazolyl)benzene) were purchased from Dojindo Lab. Ltd. First, the phosphor concentration of the liquid scintillator was optimized. The saturated solubility of Coumarin 7 in anisole was estimated to be $3 \mathrm{~g} / \mathrm{L}$. Coumarin 7 was dissolved in anisole to concentrations of $0.01,0.1,1$, and $3 \mathrm{~g} / \mathrm{L}$, and the phosphor concentration that maximizes the light yield of scintillation was investigated.

Zr-loaded green-emitting liquid scintillators were fabricated in the following manner. $\operatorname{Zr}(\mathrm{acac})_{4}$ was added to anisole solutions to concentrations of $1,3,5$, and $10 \mathrm{wt} \%$, and the solutions were allowed to stand overnight in a constant-temperature dryer (ON-300, AS ONE) at $70{ }^{\circ} \mathrm{C}$ to dissolve $\mathrm{Zr}(\mathrm{acac})_{4}$ completely in the solutions. Subsequently, optimized concentrations of Coumarin 7 were added into the anisole solutions. For comparison, Zr-loaded blue-emitting scintillators were also fabricated by dissolving DPO and POPOP to concentrations of 5 and 0.5 $\mathrm{g} / \mathrm{L}$, respectively, in the anisole solutions in accordance with a previous report. ${ }^{(6)}$

Absorption spectra of the anisole solutions were measured using a deuterium and tungsten halogen light source (DH mini, Ocean Optics). Samples were loaded into a quartz cell with a 1 $\mathrm{cm}$ optical length and placed in a sample holder (FHS-UV, Ocean Optics). Transmitted light was detected using a spectrophotometer (Flame Spectrometer; Ocean Optics) through an optical fiber (P400-2-UV-vis, Ocean Optics). Photoluminescence (PL) excitation and emission spectra of the green-emitting scintillators were measured in a quartz cell using a fluorescence spectrophotometer (F-7000, Hitachi) equipped with a $150 \mathrm{~W}$ Xe lamp as the excitation source. PL decay time profiles were measured using a fluorescence lifetime spectrometer (HORIBA, Delta Flex 3000U-TMK2). A pulsed light-emitting diode (HORIBA, NanoLED-320) with an emission wavelength of $326 \mathrm{~nm}$ was used as the excitation source. X-ray-induced radioluminescence (XRL) spectra of the green-emitting scintillators were recorded with a charge-coupled device (CCD)-based spectrometer (QE Pro Spectrometer, Ocean Insight). Sample solutions were loaded into a quartz cell with a volume of $10 \times 10 \times 9 \mathrm{~mm}^{3}$ with a polytetrafluoroethylene lid. The sample cell was attached to an optical fiber and irradiated with $\mathrm{X}$-rays from an X-ray generator (D2300-HK, Rigaku) operating at $40 \mathrm{kV}$ and $40 \mathrm{~mA}$ equipped with a $\mathrm{Cu}$ target. Pulse-height spectra were measured using gamma rays generated from ${ }^{137} \mathrm{Cs}$ to estimate the light yields of the prepared liquid scintillators. The sample cell was attached to a photomultiplier tube (PMT; R7600U-2000, Hamamatsu Photonics) using optical grease and covered with Teflon tape to collect scintillation photons. The bias voltage supplied to the PMT was set to $700 \mathrm{~V}$, and the detection signals of the PMT were amplified using a preamplifier (113 Scintillation Preamplifier, ORTEC) and a shaping amplifier (572 Amplifier, ORTEC). Amplified signals were then fed into a multichannel analyzer (MCA8000D, Amptec), and pulse-height spectra were recorded with a $500 \mathrm{~ns}$ shaping time. Scintillation decay time profiles were recorded using the delayed coincidence method. ${ }^{(22)}$ The two gamma-ray photons from the electron-positron pair annihilation caused by the beta-plus decay of ${ }^{22} \mathrm{Na}$ are generated in opposite directions. The samples and scintillation detector were simultaneously irradiated with 
$511 \mathrm{keV}$ gamma rays. To determine the timing of the gamma-ray generation, a scintillation detector consisting of a PMT (H3378-51, Hamamatsu Photonics) and a Pilot-U plastic scintillator was used. Another PMT (R7400P, Hamamatsu Photonics) was used to detect the scintillation from the samples, which was placed on the opposite side of the scintillation detector from the radiation source for time-correlated single photon counting. The distance between the R7400P PMT and the samples was adjusted to detect less than one photon for a single gamma-ray photon excitation. The detection signals were fed to a constant fraction discriminator (935 CFD, ORTEC) and converted into timing signals, which were fed into a nanosecond delay (425A, ORTEC). Start and stop signals from the scintillation detector and the R7400P PMT, respectively, were fed into a time-to-amplitude converter (566 TAC, ORTEC), and output signals from the TAC were fed into a multichannel analyzer (MCA 8000D) to obtain the scintillation decay time profiles.

\section{Results and Discussion}

\subsection{Optimization of phosphor concentration}

Figure 1 shows excitation and PL emission spectra of liquid scintillators containing different concentrations of Coumarin 7. In the sample with $0.01 \mathrm{~g} / \mathrm{L}$ Coumarin 7, a broad excitation band was observed at $350-550 \mathrm{~nm}$ and an emission peak was observed at $489 \mathrm{~nm}$. The excitation spectrum of the $0.01 \mathrm{~g} / \mathrm{L}$ Coumarin 7 solution was consistent with the absorption spectrum shown in Fig. 2. The absorption and PL emission spectra (peak wavelengths: 440 and $489 \mathrm{~nm}$, respectively) are consistent with those of Coumarin 7 in decanol solution reported in a previous study. ${ }^{(20)}$ When the phosphor concentration was increased to $0.1 \mathrm{~g} / \mathrm{L}$, the peak intensity of the excitation band decreased at $440 \mathrm{~nm}$, where the maximum absorption of Coumarin 7 was observed. It is considered that due to the high concentration of the phosphor, the excitation light was strongly absorbed on the cell surface and did not reach the inside of the sample, causing internal shielding of the emission of the phosphor and reducing the peak intensity. For the

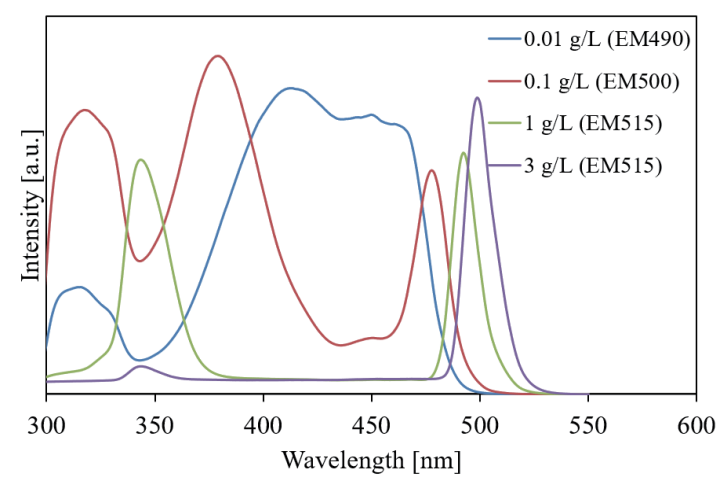

(a)

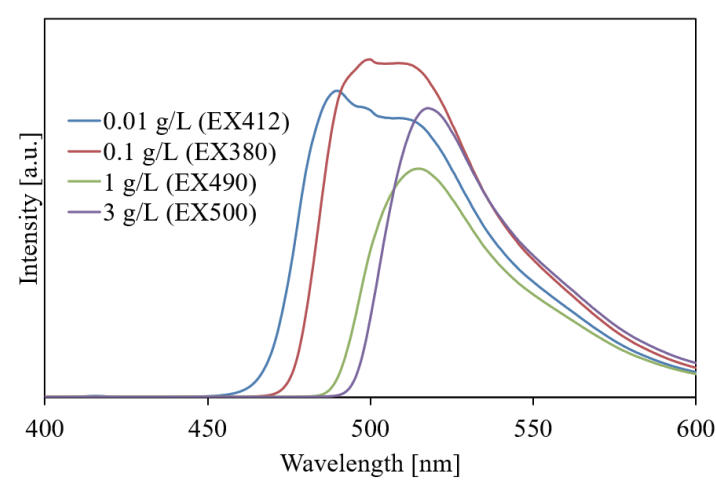

(b)

Fig. 1. (Color online) Excitation and PL emission spectra of liquid scintillators containing Coumarin 7: (a) Excitation spectra with emission wavelengths of 490, 500, 515, and $515 \mathrm{~nm}$, and (b) PL emission spectra with excitation wavelengths of 412, 380, 490, and $500 \mathrm{~nm}$ for $0.01,0.1,1$, and $3 \mathrm{~g} / \mathrm{L}$ Coumarin 7 solutions, respectively. 
phosphor concentration of $1 \mathrm{~g} / \mathrm{L}$, the two excitation peaks around 315 and $380 \mathrm{~nm}$, which were observed for $0.1 \mathrm{~g} / \mathrm{L}$ Coumarin 7 solution, disappeared owing to the internal shielding effect. Finally, in the excitation spectrum of the $3 \mathrm{~g} / \mathrm{L}$ Coumarin 7 solution, a sharp excitation peak was observed at $500 \mathrm{~nm}$, which corresponds to the long-wavelength edge of the absorption spectrum.

In the PL emission spectra, the peak wavelength redshifted from 489 to $515 \mathrm{~nm}$ with increasing concentration of Coumarin 7. It is considered that the shift is caused by the reabsorption of the emission on the short-wavelength side due to the redshift of the absorption edge of Coumarin 7 shown in Fig. 2. Figure 3 shows the XRL spectra of the liquid scintillators containing different concentrations of Coumarin 7. The XRL spectra were similar to the PL spectra, which indicates that emission from Coumarin 7 was observed. In the XRL spectra, the peak wavelength redshifted from 500 to $520 \mathrm{~nm}$, similar to in the PL emission spectra.

Figure 4 shows PL decay time profiles of the liquid scintillators containing different concentrations of Coumarin 7, in which the excitation and measurement wavelengths were 326 and $515 \mathrm{~nm}$, respectively. The decay was fitted with an exponential decay function. The decay time constants were 2.5, 2.9, 4.0, and 4.2 ns for the liquid scintillators containing Coumarin 7 at concentrations of $0.01,0.1,1$, and $3 \mathrm{~g} / \mathrm{L}$, respectively. The decay time constant of the sample with a Coumarin 7 concentration of $0.01 \mathrm{~g} / \mathrm{L}$ is consistent with that of Coumarin 7 in organic solvents reported in a previous study. ${ }^{(21)}$ The decay time constant increased from 2.5 to 4.2 ns with increasing Coumarin 7 concentration from 0.01 to $3 \mathrm{~g} / \mathrm{L}$. In the solutions having high Coumarin 7 concentrations, the emission is reabsorbed by other phosphor molecules, and the molecules are excited. Therefore, the decrease in the number of excited molecules was delayed and the PL decay time increased with the phosphor concentration.

Figure 5 shows the ${ }^{137} \mathrm{Cs}$-gamma ray pulse-height spectra of the liquid scintillators with different concentrations of Coumarin 7 and with NE-142. All the spectra were measured for the same gamma-ray photon energy, and the number of channels is proportional to the number of photons emitted during one gamma-ray photon detection event. Thus, light yields of scintillation were estimated on the basis of the relative number of channels of the Compton edge with a reference scintillator, NE-142 (light yield $=5200$ photons $/ \mathrm{MeV}$, with a volume of $5 \times 6 \times 3 \mathrm{~mm}^{3}$ ), with a correction of the quantum yields of the PMT at different scintillation wavelengths. The

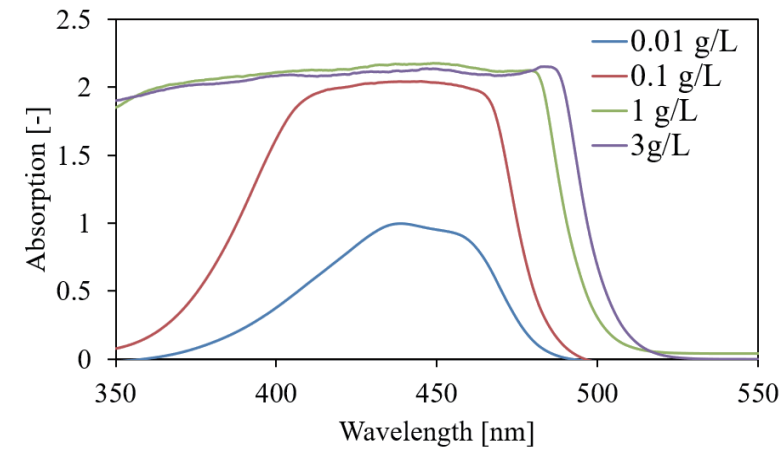

Fig. 2. (Color online) Absorption spectra of liquid scintillators containing Coumarin 7.

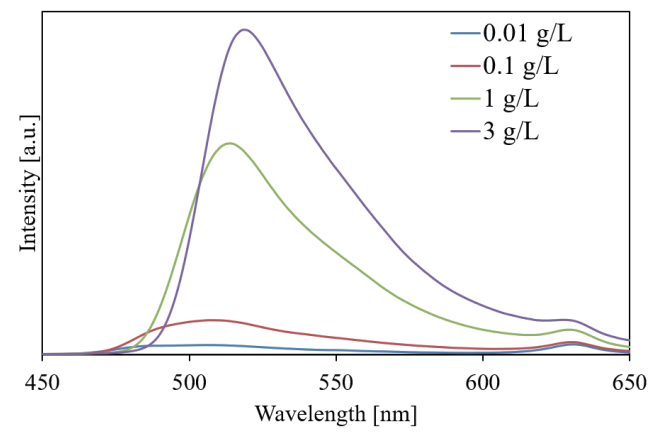

Fig. 3. (Color online) X-ray-induced radioluminescence spectra of liquid scintillators containing Coumarin 7. 


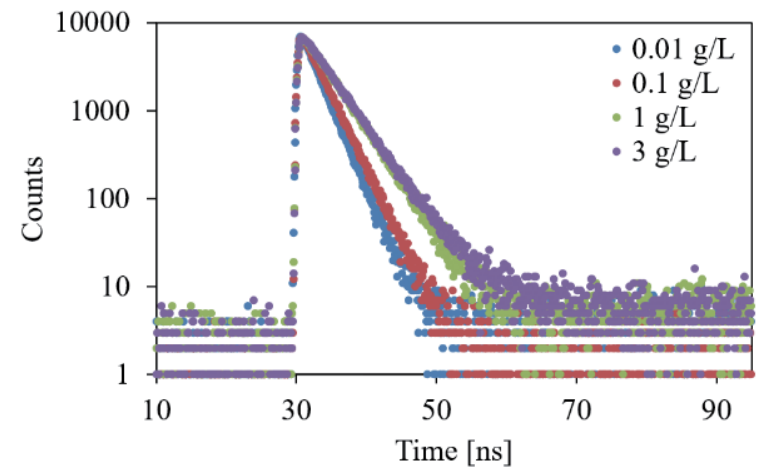

Fig. 4. (Color online) PL decay time profiles of liquid scintillators containing Coumarin 7.

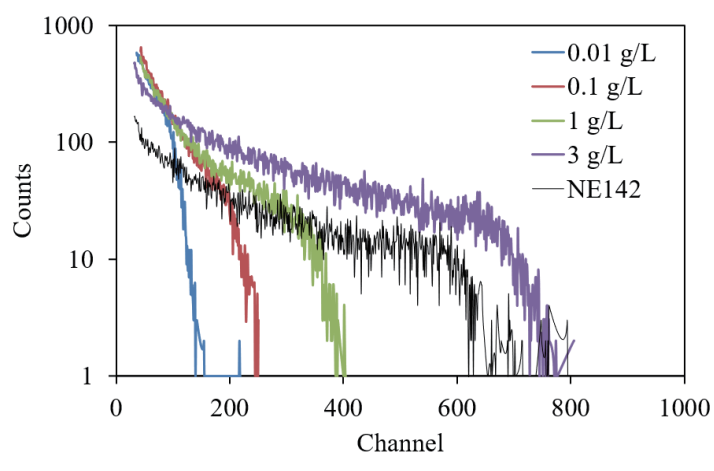

Fig. 5. (Color online) ${ }^{137} \mathrm{Cs}$-gamma ray pulse-height spectra of liquid scintillators containing Coumarin 7 and NE-142.

Table 1

Compton edge channels and light yields of liquid scintillators containing Coumarin 7 and NE-142.

\begin{tabular}{lcc}
\hline Coumarin 7 concentration & Compton edge channels & Light yield (photons/MeV) \\
\hline $0.01 \mathrm{~g} / \mathrm{L}$ & 70 & 1300 \\
$0.1 \mathrm{~g} / \mathrm{L}$ & 200 & 3700 \\
$1 \mathrm{~g} / \mathrm{L}$ & 340 & 6300 \\
$3 \mathrm{~g} / \mathrm{L}$ & 660 & 12000 \\
\hline $\mathrm{NE}-142$ & 560 & 5200 \\
\hline
\end{tabular}

estimated light yields are shown in Table 1. The highest light yield of scintillation (12000 photons/MeV) was obtained in a solution with Coumarin 7 at a concentration of $3 \mathrm{~g} / \mathrm{L}$. On the basis of the above results, the phosphor concentration used in the Zr-loaded liquid scintillators was set to $3 \mathrm{~g} / \mathrm{L}$.

\subsection{Fabrication of $\mathrm{Zr}$-loaded green-emitting liquid scintillators}

Figure 6 shows absorption spectra of anisole solutions containing $\operatorname{Zr}(\mathrm{acac})_{4}$ at different concentrations. Upon adding $1 \mathrm{wt} \% \mathrm{Zr}(\mathrm{acac})_{4}$ to anisole, the absorption edge redshifted from 300 to $340 \mathrm{~nm}$, which is attributed to the $\pi \rightarrow \pi^{*}$ transition of the acetylacetonate group coordinated to $\mathrm{Zr} .^{(17,23)}$ Upon the addition of $10 \mathrm{wt} \% \mathrm{Zr}(\mathrm{acac})_{4}$, the absorption edge shifted to $360 \mathrm{~nm}$. In addition, an increase in absorbance was observed at up to $430 \mathrm{~nm}$ in the absorption tail of $\operatorname{Zr}(\mathrm{acac})_{4}$. For typical blue-emitting liquid scintillators containing DPO and POPOP as phosphors, an emission peak is observed around $430 \mathrm{~nm} \cdot{ }^{(6,9,24)}$ Since the emission peak overlaps with the absorption tail of $\mathrm{Zr}(\mathrm{acac})_{4}$, it is considered that the light yield of scintillation markedly decreased.

Coumarin 7 was dissolved into solutions to a concentration of $3 \mathrm{~g} / \mathrm{L}$ to prepare $\mathrm{Zr}$-loaded green-emitting liquid scintillators. Figure 7 shows the excitation and PL emission spectra of the green-emitting liquid scintillators containing different concentrations of $\operatorname{Zr}(\mathrm{acac})_{4}$. In all 


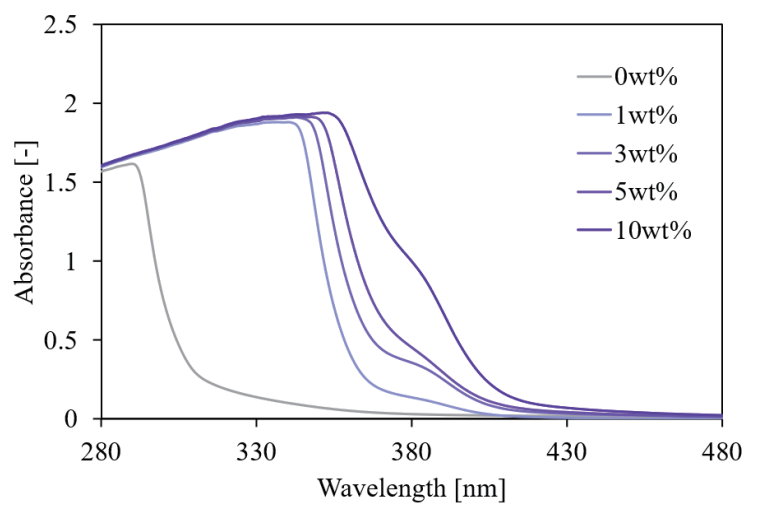

Fig. 6. (Color online) Absorption spectra of anisole solutions containing $\operatorname{Zr}(\mathrm{acac})_{4}$.

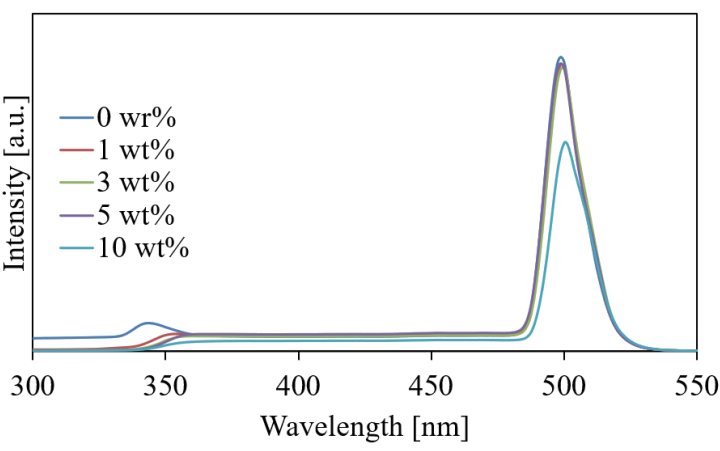

(a)

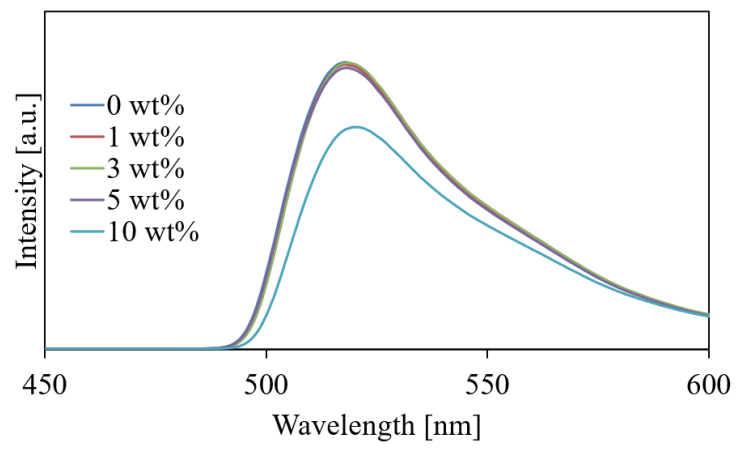

(b)

Fig. 7. (Color online) (a) Excitation (emission wavelength: $515 \mathrm{~nm}$ ) and (b) PL emission (excitation wavelength: $500 \mathrm{~nm}$ ) spectra of green-emitting liquid scintillators containing different concentrations of $\mathrm{Zr}(\mathrm{acac})_{4}$.

samples, excitation and emission peaks were observed at 500 and $515 \mathrm{~nm}$, respectively. The peaks are consistent with those of the anisole solution containing $3 \mathrm{~g} / \mathrm{L}$ of Coumarin 7 . Furthermore, the excitation intensity at wavelengths shorter than $360 \mathrm{~nm}$, where the absorption of $\mathrm{Zr}(\mathrm{acac})_{4}$ was observed in Fig. 6, was lowered by the addition of $\mathrm{Zr}(\mathrm{acac})_{4}$. It is considered that the excitation light was absorbed by $\mathrm{Zr}(\mathrm{acac})_{4}$ in this wavelength range. Furthermore, Fig. 8(a) shows the PL emission spectra excited at $270 \mathrm{~nm}$, which is the excitation wavelength of anisole. ${ }^{(6,25)}$ Similar to Fig. 7(b), the phosphor-derived emission peak was observed at $518 \mathrm{~nm}$ in all samples. These results indicate that energy transfer from anisole to Coumarin 7 occurred in the $\operatorname{Zr}(\mathrm{acac})_{4}$-added solutions. Figure $8(\mathrm{~b})$ shows XRL spectra of green-emitting liquid scintillators containing different concentrations of $\mathrm{Zr}(\mathrm{acac})_{4}$. The features of the XRL spectra of the scintillators are similar to those of the PL spectra shown in Fig 8(a), indicating that energy transfer from anisole to Coumarin 7 occurred even under X-ray excitation.

Figure 9(a) shows PL decay time profiles of the green-emitting liquid scintillators containing different concentrations of $\mathrm{Zr}(\mathrm{acac})_{4}$. The excitation and measurement wavelengths were the same as those in Fig. 4. The decay was fitted with an exponential decay function and the estimated PL decay time constants are summarized in Table 2. The PL decay time constants of 


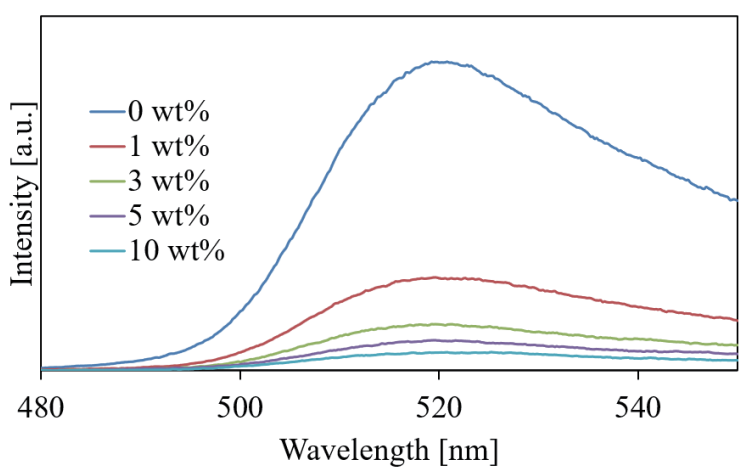

(a)

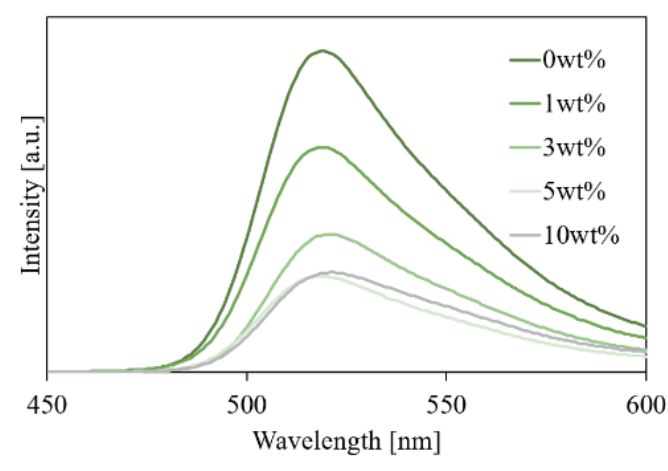

(b)

Fig. 8. (Color online) (a) PL emission spectra of green-emitting liquid scintillators containing $\operatorname{Zr}(\mathrm{acac})_{4}$ with an excitation wavelength of $270 \mathrm{~nm}$ and (b) X-ray-induced radioluminescence spectra of green-emitting liquid scintillators containing $\mathrm{Zr}(\mathrm{acac})_{4}$.

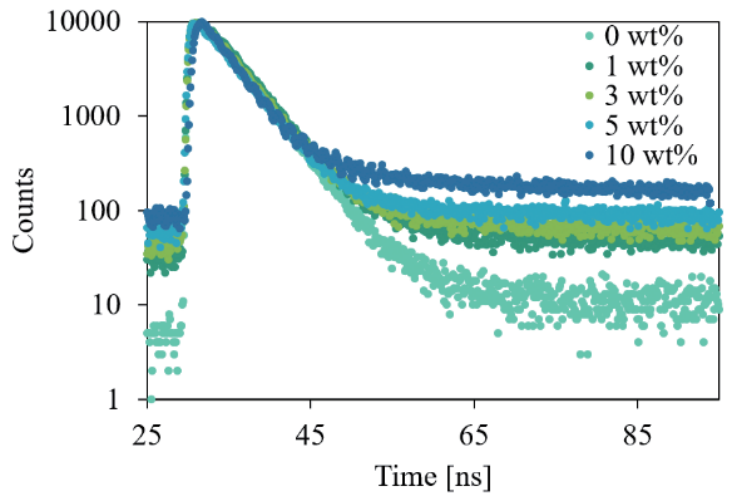

(a)

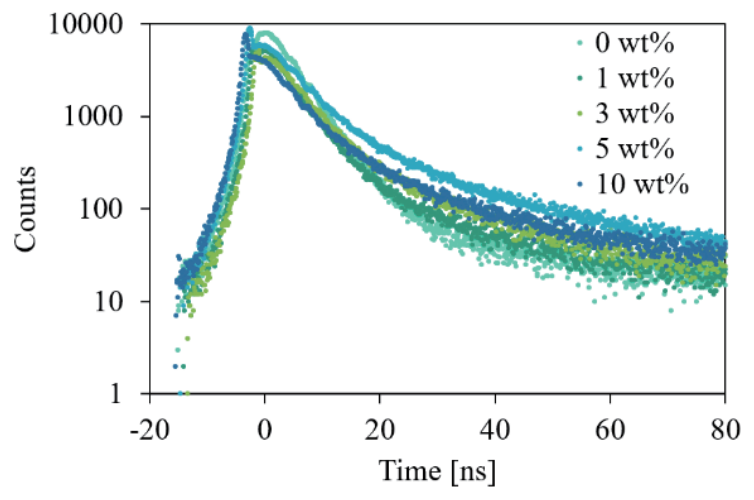

(b)

Fig. 9. (Color online) (a) PL decay time profiles and (b) $511 \mathrm{keV}$ gamma-ray scintillation decay time profiles of $\mathrm{Zr}-$ loaded green-emitting liquid scintillators containing $\mathrm{Zr}(\mathrm{acac})_{4}$.

Table 2

PL and scintillation decay time constants of green-emitting liquid scintillators containing $\mathrm{Zr}(\mathrm{acac})_{4}$.

\begin{tabular}{rccc}
\hline & PL decay time constant & \multicolumn{2}{c}{ Scintillation decay time constant } \\
\cline { 2 - 4 } & $\tau_{1}(\mathrm{~ns})$ & $\tau_{1}(\mathrm{~ns})$ & $\tau_{2}(\mathrm{~ns})$ \\
\hline $0 \mathrm{wt} \%$ & 4.5 & $4.2(96 \%)$ & $11(4 \%)$ \\
$1 \mathrm{wt} \%$ & 4.9 & $4.0(92 \%)$ & $14(8 \%)$ \\
$3 \mathrm{wt} \%$ & 4.3 & $5.1(94 \%)$ & $23(6 \%)$ \\
$5 \mathrm{wt} \%$ & 4.4 & $4.5(84 \%)$ & $16(16 \%)$ \\
$10 \mathrm{wt} \%$ & 4.3 & $4.4(83 \%)$ & $15(17 \%)$ \\
\hline
\end{tabular}

all samples were around $4.5 \mathrm{~ns}$ and are consistent with those of Coumarin 7 in anisole at a concentration of $3 \mathrm{~g} / \mathrm{L}$ estimated from Fig. 4. The PL decay time constants of the green-emitting liquid scintillators were not affected by the addition of $\operatorname{Zr}(\text { acac })_{4}$. Figure 9(b) shows scintillation decay time profiles of the green-emitting liquid scintillators containing different concentrations of $\operatorname{Zr}(\mathrm{acac})_{4}$. The decay was fit using a sum of two exponential decay functions to obtain the 
scintillation decay time constants (Table 2). Responses on the nanosecond scale were confirmed for all liquid scintillators with $\operatorname{Zr}(\mathrm{acac})_{4}$. The scintillation decay time constants of the fast component of all samples were around $4.4 \mathrm{~ns}$, consistent with the PL decay time constants of the $3 \mathrm{~g} / \mathrm{L}$ Coumarin 7 solutions. In the scintillation decay time profiles, a long component of about 16 ns, which did not appear in the PL decay, was observed. The component is attributed to a delayed emission from the phosphor molecules of the singlet state due to the interaction between the two triplet excitations. ${ }^{(26)}$ In addition, the contribution of the long component increased from 4 to $17 \%$ with increasing $\mathrm{Zr}(\mathrm{acac})_{4}$ concentration.

Figure 10(a) shows the ${ }^{137} \mathrm{Cs}$-gamma ray pulse-height spectra of the green-emitting liquid scintillators containing different concentrations of $\mathrm{Zr}(\mathrm{acac})_{4}$ and containing NE-142. For comparison, the pulse-height spectra of the blue-emitting liquid scintillators containing different concentrations of $\mathrm{Zr}(\mathrm{acac})_{4}$ are shown in Fig. 10(b). Light yields of scintillation were estimated by the same procedure as in Sect. 3.1. Table 3 summarizes the light yields of the green-emitting and blue-emitting liquid scintillators containing different concentrations of $\operatorname{Zr}(\mathrm{acac})_{4}$ and

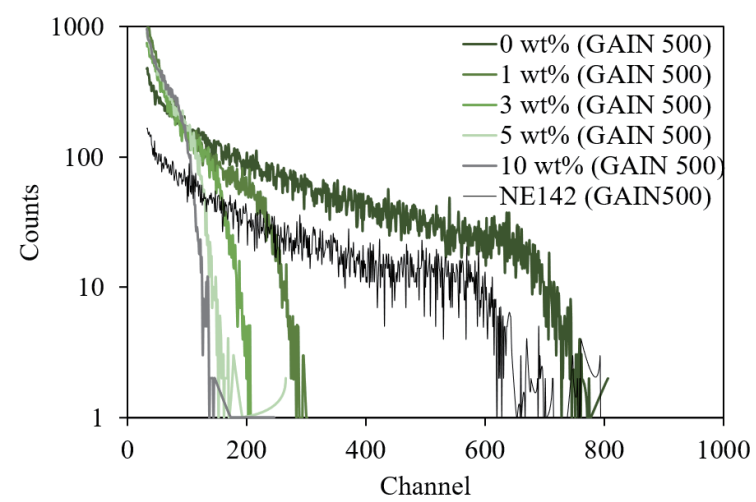

(a)

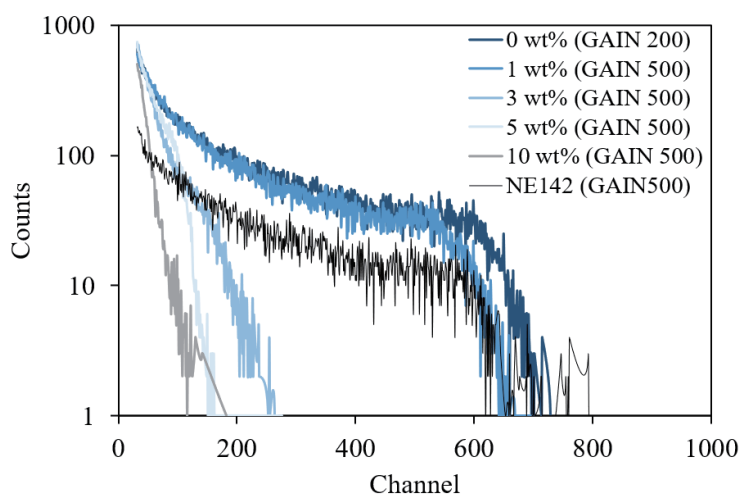

(b)

Fig. 10. (Color online) ${ }^{137}$ Cs-gamma ray pulse-height spectra of (a) green-emitting and (b) blue-emitting liquid scintillators containing $\mathrm{Zr}(\mathrm{acac})_{4}$ and NE-142.

Table 3

Compton edge channels and light yields of green-emitting and blue-emitting liquid scintillators containing different concentrations of $\mathrm{Zr}(\mathrm{acac})_{4}$ and containing NE-142.

\begin{tabular}{lccc}
\hline & $\begin{array}{c}\text { Zr(acac) } \\
\text { concentration }(w t \%)\end{array}$ & $\begin{array}{c}\text { Compton edge } \\
\text { channels }\end{array}$ & $\begin{array}{c}\text { Light yield } \\
\text { (photons/MeV) }\end{array}$ \\
\hline Green-emitting & 0 & 660 & 12000 \\
scintillator & 1 & 210 & 3900 \\
& 3 & 140 & 2600 \\
& 5 & 100 & 1900 \\
Blue-emitting & 10 & 100 & 1900 \\
scintillator & 0 & 1500 & 14000 \\
& 1 & 530 & 4900 \\
& 3 & 140 & 8300 \\
NE-142 & 5 & 90 & 370 \\
\hline
\end{tabular}


containing NE-142. Quenching of scintillation by $\operatorname{Zr}(\mathrm{acac})_{4}$ was observed in both the blue- and green-emitting liquid scintillators. In the blue-emitting liquid scintillator, the light yield decreased from 14000 to 370 photons $/ \mathrm{MeV}$ when the concentration of $\operatorname{Zr}(\mathrm{acac})_{4}$ was increased from 0 to $10 \mathrm{wt} \%$. On the other hand, in the green-emitting liquid scintillator, the light yield decreased from 12000 to 1900 photons/MeV. There was no significant difference in the light yields between samples with 5 and $10 \mathrm{wt} \% \mathrm{Zr}(\mathrm{acac})_{4}$ concentrations. The decrease in the light yield of the green-emitting scintillator is consistent with the decrease in the emission intensity of XRL spectra shown in Fig. 8(b). Comparison of the samples with $\mathrm{Zr}(\mathrm{acac})_{4}$ concentration of 10 wt $\%$ shows that the light yield of the green-emitting liquid scintillator was more than five times that of the blue-emitting liquid scintillator. The results of this study show that high-concentration $\mathrm{Zr}$-containing liquid scintillators having high light yields can be fabricated with Coumarin 7 as a phosphor.

\section{Conclusions}

A candidate isotope-loaded green-emitting liquid scintillator using Coumarin 7 was developed for the detection of neutrinoless double beta decay events. First, the concentration of Coumarin 7 was optimized. The highest light yield of 12000 photons $/ \mathrm{MeV}$ was obtained for an anisole solution containing $3 \mathrm{~g} / \mathrm{L}$ of Coumarin 7. A scintillation peak in the XRL spectrum was observed at $515 \mathrm{~nm}$, which does not overlap with the absorption of $\mathrm{Zr}(\mathrm{acac})_{4}$. Second, $\mathrm{Zr}(\mathrm{acac})_{4}$ was dissolved in the green-emitting liquid scintillator. The light yield of the green-emitting liquid scintillator containing $10 \mathrm{wt} \% \mathrm{Zr}(\mathrm{acac})_{4}$ was 1900 photons/ $\mathrm{MeV}$, which was higher than that of 370 photons $/ \mathrm{MeV}$ for the blue-emitting liquid scintillator with the same $\mathrm{Zr}(\mathrm{acac})_{4}$ concentration. Furthermore, the decrease in the light yield was suppressed at concentrations of 5 $\mathrm{wt} \% \mathrm{Zr}(\mathrm{acac})_{4}$ and above. By producing liquid scintillators that emit at a longer wavelength, the absorption of $\mathrm{Zr}(\mathrm{acac})_{4}$ was avoided, which results in an improvement in the light yield. The scintillation decay time constants of the fast component were around $4.4 \mathrm{~ns}$. As a result, the proposed protocol with Coumarin 7 allows the fabrication of liquid scintillators with high light yields that are loaded with $10 \mathrm{wt} \% \mathrm{Zr}(\mathrm{acac})_{4}$.

\section{Acknowledgments}

This research was supported by JSPS KAKENHI Grant Numbers 18H03890 and 20K20917. Part of this research is based on the Cooperative Research Project of the Research Center for Biomedical Engineering, Ministry of Education, Culture, Sports, Science and Technology.

\section{References}

1 J. Schechter and J. W. F. Valle: Phys. Rev. D 25 (1982) 2951.

2 J. D. Vergados, H. Ejiri, and F. Šimkovic: Int. J. Mod. Phys. E 25 (2016) 1630007.

3 S. Dell'Oro, S. Marcocci, M. Viel, and F. Vissani: Adv. High Energy Phys. 2016 (2016) 2162659.

4 K. Nakamura: Nucl. Phys. B 229-232 (2012) 128.

5 C. Buck and M. Yeh: J. Phys. G 43 (2016) 093001.

6 Y. Fukuda, S. Moriyama, and I. Ogawa: Nucl. Instrum. Methods Phys. Res., Sect. A 732 (2013) 397. 
7 V. M. Gehmen, P. J. Doe, R. G. H. Robertson, D. I. Will, H. Ejiri, and R. Hazama: Nucl. Instrum. Methods Phys. Res., Sect. A 622 (2010) 602.

8 M. J. Hwang, Y. J. Kwon, H. J. Kim, J. W. Kwak, S. C. Kim, S. K. Kim, T. Y. Kim, S. Y. Kim, H. S. Lee, M. J. Lee, S. S. Myung, Y. D. Kim, J. I. Lee, W. G. Kang, I. S. Hahn, and M. H. Lee: Astropart. Phys. 31 (2009) 412.

9 I. B. Nemchenok, V. B. Brudanin, O. I. kochetov. V. V. Timkin, and A. A. Shurenkova: Bull. Russ. Acad. Sci. Phys. 75 (2011) 1007.

10 S. Joung, Y. Kim, J. Kim, J. Park, M. Jang, J. Lee, C. Kim, M. S. Lee, and J. Lim: Appl. Radiat. Isot. 170 (2021) 109593.

11 M. Apollonio, A. Baldini, C. Bemporad, E. Caffau, F. Cei, Y. Déclais, H. de Kerret, B. Dieterle, A. Etenko, L. Foresti, J. George, G. Giannini, M. Grassi, Y. Kozlov, W. Kropp, D. Kryn, M. Laiman, C. E. Lane, B. Lefiévre, I. Machulin, A. Martemyanov, V. Martemyanov, L. Mikaelyan, D. Nicolò, M. Obolensky, R. Pazzi, G. Pieri, L. Price, S. Riley, R. Reeder, A. Sabelnikov, G. Santin, M. Skorokhvatov, H. Sobel, J. Steele, R. Steinberg, S. Sukhotin, S. Tomshaw, D. Veron, and V. Vyrodov: Eur. Phys. J. C 27 (2003) 331.

12 A. R. Ronzio, C. L. Cowan, and F. Reines: Rev. Sci. Instrum. 26 (1958) 146.

13 R. S. Raghavan: Phys. Rev. Lett. 37 (1976) 259.

14 Y. Ding, Z. Zhang, J. Liu, Z. Wang, P. Zhou, and Y. Zhao: Nucl. Instrum. Methods Phys. Res., Sect. A 584 (2008) 238.

15 C. Buck, F. X. Hartmann, D. Motta, and S. Schoenert: Chem. Phys. Lett. 435 (2007) 252.

16 Y. Fukuda, T. Gunji, S. Moriyama, and I. Ogawa: Nucl. Part. Phys. Proc. 273-275 (2016) 2615.

17 A. Potdevin, V. Briois, N. Caperaa, C. V. Santilli, G. Chadeyron, and R. Mahiou: RSC Adv. 6 (2016) 41962.

18 U. R. Raikar, C. G. Renuka, Y. F. Nadaf, B. G. Mulimani, A. M. Karguppikar, and M. K. Soudagar: Spectrohim. Acta, Part A 65 (2006) 673.

19 A. Satpati, S. Senthilkumar, M. Kumbhakar, S. Nath, D. K. Maity, and H. Pal: Photochem. Photobiol. 81 (2005) 270278.

20 A. K. Satpati, M. Kumbhakar, S. Nath, and H. Pal: Photochem. Photobiol. 85 (2009) 119.

21 G. B. Dutt and S. Raman: J. Chem. Phys. 114 (2001) 6702.

22 L. M. Bollinger and G. E. Thomas: Rev. Sci. Instrum. 32 (1961) 1044.

23 M. Veith: J. Sol-Gel Sci. Technol. 46 (2008) 291.

24 S. Arai, M. Koshimizu, Y. Fujimoto, T. Yanagida, and K. Asai: Nucl. Instrum. Methods Phys. Res., Sect. A 954 (2020) 161632.

25 S. Faust, T. Dreier, and C. Schulz: Appl. Phys. B 112 (2013) 203.

26 J. B. Briks: Chem. Phys. Lett. 7 (1970) 293. 